\section{FDA approves first-in-class cancer metabolism drug}

The FDA approved Agios' and Celgene's enasidenib for acute myeloid leukaemia (AML), validating metabolism-modulating drugs as a means of killing cancer cells.

Enasidenib (formerly AG-221) is a first-in-class inhibitor of mutated isocitrate dehydrogenase 2 (IDH2). The IDH enzymes normally metabolize isocitrate into $\alpha$-ketoglutarate. When they are mutated in cancers, they also convert $\alpha$-ketoglutarate into 2-hydroxyglutarate, an oncometabolite that causes cell differentiation defects by impairing histone demethylation.

In clinical trials of enasidenib, $23 \%$ of treated patients had complete responses or complete responses with partial haematologic recovery lasting a median of 8.2 months. The most common side effects were nausea, vomiting, diarrhoea, elevated bilirubin and decreased appetite. The agency approved the drug with a black box warning noting the risk of differentiation syndrome, a potentially fatal complication that is associated with certain forms of AML.

The FDA also approved a companion diagnostic alongside the drug to detect IDH2 mutations.

Agios discovered and developed the drug in just 8 years. The company started working on IDH2 inhibitors in 2009 and advanced its lead candidate into clinical trials in 2013.

Although the approval highlights the promise of metabolism-modulating anticancer drugs, researchers have struggled to find other therapeutically tractable targets in metabolic pathways (Nat. Rev. Drug Discov. 15, 735-737; 2016). Only a handful of other cancer metabolism candidates have made it into clinical trials. Agios' IDH1 inhibitor is in phase III trials in AML and bile duct cancer. Calithera Biosciences' glutaminase 1 inhibitor CB-839 is in phase II trials for renal cell cancer.

Several of the companies that started in the cancer metabolism space have turned their focus to immunometabolism-modulating drugs that can improve immune cell survival or modify the interactions between cancer cells and immune cells in the microenvironment. Incyte's phase III indoleamine 2,3-dioxygenase 1 (IDO1) inhibitor epacadostat, for example, controls tryptophan metabolism to foster immune cell activity.

Asher Mullard

\section{Diabetes drug shows promise in Parkinson disease}

There are no approved disease-modifying drugs for Parkinson disease, a

neurodegenerative condition that affects $2-3 \%$ of people aged $\geq 65$ years. Accumulating evidence suggests that GLP1 receptor agonists - incretin mimetics that researchers first developed for the treatment of diabetes may have neuroprotective properties. A first randomized, placebo-controlled trial of AstraZeneca's GLP1 receptor agonist exenatide now provides further support for these agents as possible Parkinson drugs, researchers report in The Lancet.

The team of academic researchers ran a phase II trial of exenatide in 62 Parkinson patients, looking at the drug's effects on the 132-point Movement Disorders Society Unified Parkinson Disease Rating Scale (MDS-UPDRS) part 3 motor function score. After 60 weeks, there was a statistically significant 3.5 point adjusted difference between the exenatide and the placebo groups. Both groups experienced injection site reactions and gastrointestinal symptoms as common adverse events.
These findings are in line with a previous open-label trial of exenatide in Parkinson disease, run by the same researchers. "Whether this drug acts as a novel symptomatic agent, influences compensatory responses or behaviours, or has neuroprotective effects on underlying pathology is unclear, but there is a strong indication that GLP1 receptor agonists may have a useful role in future treatment of Parkinson disease," they write. They add that the role of these drugs is also being explored in other neurodegenerative diseases, including Alzheimer disease, amyotrophic lateral sclerosis and multiple sclerosis.

In a linked commentary, neurologists from the Medical University Innsbruck call the findings exciting but note several caveats. The baseline characteristics of the treatment and placebo groups were unbalanced; exenatide-treated patients tended to be older, for example, and had higher starting MDS-UPDRS part 3 scores. Exenatide-treated patients had greater increases in their concomitant use of dopaminergic therapy during the trial. And, the trial did not find significant improvements in any of the secondary outcome measures.
The trial was funded by the Michael J. Fox Foundation for Parkinson's Research. There are as yet no industry-sponsored trials of GLP1 receptor agonists in Parkinson disease. Earlier this year, a few companies advanced the first $\alpha$-synuclein-targeted agents into phase II trials (Nat. Rev. Drug Discov. 16, 371-373; 2017).

Asher Mullard

\section{FDA rejects first-in-class osteoporosis drug}

\section{The FDA rejected Amgen's and UCB's} romosozumab, an anti-sclerostin monoclonal antibody that the firms are developing for the treatment of osteoporosis. The rejection followed on the heels of a cardiovascular safety signal that the companies reported in May. Amgen and UCB are now working to resubmit the application and hope to incorporate new data from ongoing phase III trials to address regulatory questions and concerns.

Nearly all of the osteoporosis drugs that have been approved to date act by lowering bone resorption processes, but in doing so they also reduce bone-rebuilding activity. The one exception is Eli Lilly's teriparatide, which boosts bone formation but also increases bone resorption. Researchers hoped that romosozumab might become the first drug that would decouple bone formation from bone resorption; the drug increases bone formation while simultaneously lowering bone resorption markers (Nat. Rev. Drug Discov. 15, 445-446; 2016).

The anti-sclerostin programme started in the 1990s and was spurred on by human genetic data that showed that loss-of-function mutations in the gene that encodes sclerostin leads to high bone mineral density without other adverse events (Nat. Rev. Drug Discov. 16, 515-518; 2017). Clinical data supported the growing enthusiasm, with treatment leading to a $73 \%$ reduction in the relative risk of spine fracture at 12 months in a first pivotal phase III trial in postmenopausal women with osteoporosis. In May, however, the company reported that a second phase III trial found a $30 \%$ increase in cardiovascular events with romosozumab treatment. The companies are still working to understand the safety signal.

At least one other anti-sclerostin drug is in clinical trials: in May, Mereo BioPharma initiated a phase II trial of the BPS-804 antibody in patients with osteogenesis imperfecta, a genetic condition that is also known as brittle bone disease.

Asher Mullard 